METABOLIC PATHWAYS

\section{Sunny side up}

Cell 173, 1-12 (2018)

Did you ever feel better, sharper after a little time outside? Well, you are not alone. Moderate doses of sunshine have been linked to improved emotional and learning states, but the 'how' for these effects has remained largely unexplained. UV-irradiation does lead to changes in levels of small and large molecules in the skin. In a new study, investigators linked one such metabolite, urocanic acid (UCA), to these beneficial effects.

Using new single neuron metabolomics and isotopic labeling, authors reported that UCA was metabolized to glutamic acid (GLU), the most common excitatory neurotransmitter in mammals, in several regions of the brain. Moreover, increases in UCA-derived GLU improved memory and learning. This metabolic pathway was not previously known to exist in neural tissues.

https://doi.org/10.1038/s41684-018-0104-X

\section{AGEING}

\section{DNA repair and longevity}

elife, https://doi.org/10.7554/eLife.34836 (2018)

To date, studies have indicated a correlation between the extent of DNA repair and longevity, but a mechanism was lacking. Moreover, aging is often associated with metabolic dysregulation. A new study that worked with two in vivo models firms this up, establishing a molecular link between lifespan, DNA repair, and metabolism. When DNA damage repair (DDR) was activated in Caenorhabditis elegans by treatment with moderate doses of chloroquine, a DDR activator, worms lived longer, but not worms deficient in the ataxia telangiectasia mutated (ATM) protein, a kinase and regulatory enzyme involved in DDR. Additionally, mice modeling Hutchinson-Gilford progeria syndrome, a genetic disorder that results in a significant reduction in longevity, maintained on chloroquine enjoyed extended lifespan and healthspan. Finally, investigators demonstrated that ATM promotes SIRT6, a histone deacetylase associated with longevity and metabolism, stability and thereby function.

\section{GENOME}

\section{New bat genome and immunity \\ Cell 173, 1098-1110 (2018)}

The Egyptian rousette (Rousettus aegyptiacus) is the only known reservoir for Marburg virus, a lethal filovirus that results in hemorrhagic fever. This bat's genome was just analyzed using a combination of short and long reads to provide a 169x coverage, with an estimated size of $2 \mathrm{~Gb}$. Analysis of the sequence revealed that loci associated with immunity were increased in comparison to other mammals, including: histocompatibility complex I loci, natural killer cell receptors, and interferons.

Furthermore, the data point towards a more nuanced response to viral infections in bats. Other studies suggest that bat species' broad resistance to human viruses is possibly the result of a strong innate immune response. However, this work indicates that elevated tolerance of infection with reduced inflammatory responses is more likely the source, at least in the Egyptian rousette. $\mathrm{CN}$ https://doi.org/10.1038/s41684-018-0106-8

\section{BIOLOGICAL MODELS}

\section{Termites and aging \\ PNAS 115, 5504-5509 (2018)}

Within one colony, social hymenopteran lifespans can span more than a hundred-fold difference between caste members. In a new study with termites, investigators compared reproductive caste members (kings and queens) to working caste members (majors and minors) using transcriptome sequencing of head tissues. They discovered that majors, who typically have shorter lives due to performing outside duties, showed elevated expression of transposable elements (TE) and TE-related genes in old majors versus young majors. Alternatively, genes known to suppress TE, termed PIWI-interacting RNAs, were reduced in old majors. Reports from other organisms also link elevated TE activity to increased aging, with TE believed to randomly insert into genes and regulatory regions, thereby disrupting function. The authors liken a colony to a superorganism, where the majors are the disposable soma that is not maintained due to its' reduced longevity.

https://doi.org/10.1038/s41684-018-0107-7

Ellen P. Neff, Clark Nelson

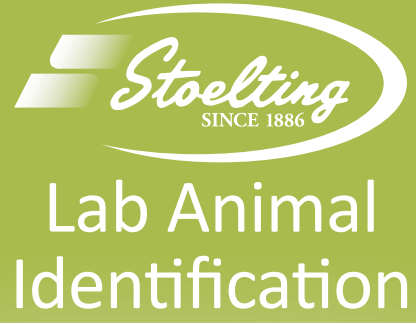

SMALL ANIMAL EARTAGS AND MARKERS
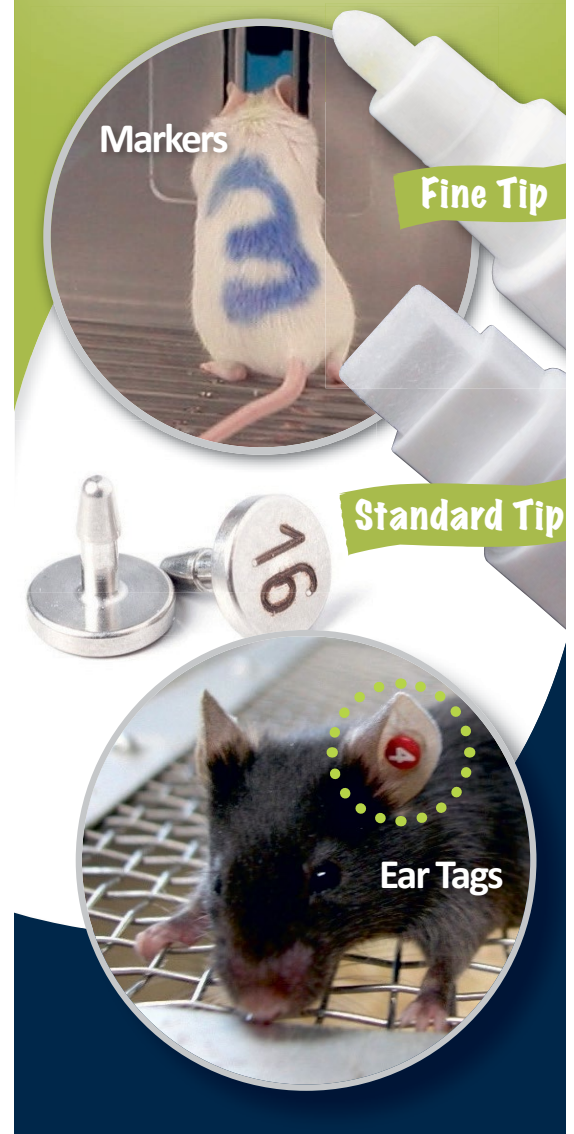

Ear Tag and Marker Features:

- Quick and easy identification

- Long-lasting

- Nontoxic, non-hazardous

- Multiple colors options available

Contact Stoelting for details! Visit

www.StoeltingCo.com or email us at info@StoeltingCo.com 